\title{
Group I p21-activated kinases regulate thyroid cancer cell migration and are overexpressed and activated in thyroid cancer invasion
}

\author{
Samantha K McCarty ${ }^{1}$, Motoyasu Saji ${ }^{1}$ Xiaoli Zhang ${ }^{2}$, David Jarjoura ${ }^{2}$, \\ Alfredo Fusco ${ }^{3}$, Vasyl V Vasko ${ }^{4}$ and Matthew D Ringel ${ }^{1}$ \\ ${ }^{1}$ Divisions of Endocrinology and Oncology and ${ }^{2}$ Center for Biostatistics, The Ohio State University College of Medicine and \\ Arthur G. James Comprehensive Cancer Center, 1581 Dodd Drive, 445D, Columbus, Ohio 43210, USA \\ ${ }^{3}$ Instituto di Endocrinologia ed Oncologia Sperimentale del CNR c/o Dipartimento di Biologia e Patologia Cellulare e Molecolare, \\ Università di Napoli 'Federico II', Via Pansini 5, Naples 80138, Italy \\ ${ }^{4}$ Uniformed Services University of Health Science, Bethesda, Maryland 20814, USA
}

(Correspondence should be addressed to M D Ringel; Email: matthew.ringel@osumc.edu)

\begin{abstract}
p21-activated kinases (PAKs) are a family of serine/threonine kinases that regulate cytoskeletal dynamics and cell motility. PAKs are subdivided into group I (PAKs 1-3) and group II (PAKs 4-6) on the basis of structural and functional characteristics. Based on prior gene expression data that predicted enhanced PAK signaling in the invasive fronts of aggressive papillary thyroid cancers (PTCs), we hypothesized that PAKs functionally regulate thyroid cancer cell motility and are activated in PTC invasive fronts. We examined PAK isoform expression in six human thyroid cancer cell lines (BCPAP, KTC1, TPC1, FTC133, C643, and SW1746) by quantitative reverse transcription-PCR and western blot. All cell lines expressed PAKs 1-4 and PAK6 mRNA and PAKs 1-4 protein; PAK6 protein was variably expressed. Samples from normal and malignant thyroid tissues also expressed PAKs 1-4 and PAK6 mRNA; transfection with the group I (PAKs 1-3) PAK-specific p21 inhibitory domain molecular inhibitor reduced transwell filter migration by $\sim 50 \%$ without altering viability in all cell lines $(P<0.05)$. BCPAP and FTC133 cells were transfected with PAK1, PAK2, or PAK3-specific small interfering RNA (siRNA); only PAK1 siRNA reduced migration significantly for both cell lines. Immunohistochemical analysis of seven invasive PTCs demonstrated an increase in PAK1 and pPAK immunoactivity in the invasive fronts versus the tumor center. In conclusion, PAK isoforms are expressed in human thyroid tissues and cell lines. PAK1 regulates thyroid cancer cell motility, and PAK1 and PPAK levels are increased in PTC invasive fronts. These data implicate PAKs as regulators of thyroid cancer invasion.
\end{abstract}

Endocrine-Related Cancer (2010) 17 989-999

\section{Introduction}

Thyroid cancer incidence is increasing, and it is estimated that $\sim 37000$ new cases of thyroid cancer will be diagnosed and $\sim 1500$ patients will die from this disease in 2009 (American Cancer Society; http:// www.cancer.org). Over the past several years, there has been intense interest in developing new therapies for patients with thyroid cancers that progress despite standard treatments (Ringel 2009). Encouraging results have been published from clinical trials using kinase inhibitors with partial remission rates $\sim 30 \%$ and even higher rates of progression-free survival (Schlumberger \& Sherman 2009). One common target of the compounds thought to be critical to their activities is the family of vascular endothelial growth factor (VEGF) receptors. However, each of these compounds inhibits other kinases, including thyroidrelated oncogenes such as Ret kinase and BRAF (Schlumberger \& Sherman 2009). While the precise targets of action responsible for clinical activity are 
uncertain, responses are not complete or durable, emphasizing the need to further research-defining mechanisms of thyroid cancer progression.

Predictors of poor survival from thyroid cancer include anaplastic histology, distant metastases, and gross local invasion of the primary tumor (Leboulleux et al. 2005, Pulcrano et al. 2007). We previously analyzed the invasive fronts of grossly invasive papillary thyroid cancers (PTCs), and demonstrated that the invasive regions have a mRNA expression profile consistent with epithelial-to-mesenchymal transition (EMT) with overrepresentation of transforming growth factor- $\beta$ (TGF- $\beta$ ) integrin and small GTPase/ p21-activated kinase (PAK) signaling (Vasko et al. 2007). EMT is a process in which there is remodeling of the cytoskeleton and loss of cell contact inhibition of growth leading to enhanced cellular capacity to migrate and invade, which is thought to be involved in cancer progression (Vasko \& Saji 2007, Kalluri \& Weinberg 2009, Tsuji et al. 2009). Hallmarks of EMT include increased expression and organization of intermediate filaments, increased expression of vimentin and osteopontin, loss of membranous $\beta$-catenin, and loss of E-cadherin expression (Kalluri \& Weinberg 2009). Functional mediators of EMT are TGF- $\beta$,

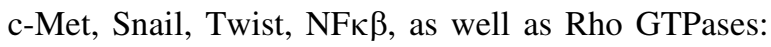
RhoA, Cdc42, and Rac (Thiery 2002). In PTC, overexpression of vimentin was shown to be independently associated with increased tumor invasiveness and nodal metastases (Vasko et al. 2007).

PAKs are direct downstream targets of $\mathrm{Cdc} 42$ and Rac, which organize actin and intermediate filaments, enhance growth, and inhibit apoptosis (Jaffer \& Chernoff 2002, Bokoch 2003, Vadlamudi \& Kumar 2003, Kumar et al. 2006, Zhao et al. 2006). There are six isoforms of PAK which are divided into two families, group I (PAKs 1-3; Bokoch 2003) and group II (PAKs 4-6; Jaffer \& Chernoff 2002) based on structural and functional similarities. Group I PAKs exist in an inactive homodimer maintained by interactions between the autoinhibitory domain (AID) and kinase domain of PAK monomers (Kumar \& Vadlamudi 2002). Upon binding to Cdc42 or Rac, the dimeric structure relaxes allowing for functional phosphorylation by phosphoinositide dependent kinase 1 (PDK1), AKT, and other PAK molecules. Two phosphorylation sites, ser144 and thr423 (In PAK1), are particularly important for PAK activity (Bokoch 2003). Group I PAKs can also be activated by nonGTPase mechanisms (Bokoch 2003, Zhao \& Manser 2005), and they serve as scaffolds for SH3 domaincontaining proteins (Bokoch 2003, Higuchi et al. 2008). Group II PAKs also bind Rac and Cdc42, but they lack an AID, exist as active monomers, and have not been reported to have a scaffolding function (Jaffer \& Chernoff 2002).

PAKs regulate actin and intermediate fiber dynamics directly by phosphorylating targets such as vimentin, desmin, LIM kinase, myosin light chains, and myosin light chain kinase (Bokoch 2003, Zhao \& Manser 2005). PAK also regulates the function of other EMTrelated targets including TGF- $\beta$ (18-20), Snail, filamin $(21,22), \mathrm{NF} \kappa \beta$ (Frost et al. 2000), and NF2. Finally, PAK1 is a breast cancer oncogene in vivo, has been associated with more aggressive clinical features, and is functionally involved in the development of tamoxifen resistance in estrogen receptor-positive breast cancer cells (Holm et al. 2006, Rayala et al. 2006). Thus, based on the gene expression arrays in PTC invasive fronts and the known functions of PAK in other systems, we hypothesized that PAKs are functionally important in thyroid cancer.

\section{Materials and methods}

\section{Human thyroid tissues and cell lines}

The Institutional Review Boards of the Ohio State University and Uniformed Services University of the Health Sciences approved sample collection, and patient consents were obtained. A total of 11 samples were obtained; 4 frozen-paired normal and tumor samples for gene expression studies and 7 paraffinembedded samples from locally invasive thyroid cancers for immunohistochemistry (IHC).

Human carcinoma BCPAP, KTC1, TPC1, FTC133, C643, and SW1746 cell lines were the generous gifts of Drs Rebecca Schweppe and Bryan Haugen (University of Colorado, Denver, CO, USA; Schweppe et al. 2008) with permission from the researchers who originally established cell lines: BCPAP - Dr Nicole Fabien, Centre Hospitalier Lyon-Sud, France (Fabien et al. 1994); KTC1 - Dr Junichi Kurebayashi, Kawasaki Medical School, Japan (Kurebayashi et al. 2000): TPC1 - Dr Hiroshi Sato, Kanazawa University, Japan (established by the late Dr Nobuo Sato (Tanaka et al. 1987)); FTC133 - Dr Peter E Goretzki, University of Leipzig, Germany (Goretzki et al. 1990); and C643 and SW1736 - Dr Nils-Erik Heldin, University Hospital, Uppsala, Sweden (Gustavsson et al. 1996, Xu et al. 2003). The obtained clones were all confirmed to be of thyroid origin by DNA fingerprinting using methods previously described (Schweppe et al. 2008). These cell lines are representative of the three primary types of thyroid cancer and express common thyroid oncogenes 
including BRAF V600E, mutated RAS, or RET/PTC or to have loss of PTEN expression (Supplementary Table 1, see section on supplementary data given at the end of this article). BCPAP, KTC1, C643, and SW1746 were grown in RPMI 1640 medium (Life Technologies Co.), while FTC133 and TPC1 were grown in DMEM (Life Technologies Co.), both supplemented with $10 \%$ fetal bovine serum (FBS) and non-essential amino acids (NEAA) unless as noted for experiments.

\section{Transient transfections and plasmids}

Vectors containing cDNAs encoding each of the six PAK isoforms and green fluorescent protein (GFP)tagged PAK inhibitory domain (PID) vector were the generous gifts of Dr Jonathan Chernoff (Fox Chase Cancer Centre; Beeser \& Chernoff 2005). Each PAK isoform cDNA was transfected into HEK293 cells using Lipofectamine and Plus reagents (Life Technologies Co.), and RNA and protein were isolated using methods described in detail below. In preliminary experiments with the thyroid cancer cell lines, PAK phosphorylation was noted to be diminished as cells became confluent (data not shown); thus, for PAK inhibition, transfections were performed using Optifect Reagent (Life Technologies Co.) when cells were $30-40 \%$ confluent. After 4 h of incubation, the liposomes were aspirated; either RPMI 1640 medium or DMEM supplemented with 10\% FBS (Life Technologies Co.) was added to the cells for $24 \mathrm{~h}$ before performing migration assays. PID transfection efficiency was estimated by visualizing the percentage of GFPexpressing cells using fluorescence microscopy.

\section{SiRNA transfections}

BCPAP and FTC133 cells were grown to $30-40 \%$ confluency and transfected with scrambled control (cat. sc-37007 Santa Cruz Biotechnology, Inc., Santa Cruz, CA, USA) or PAK1, PAK2, and PAK3-pooled specific siRNAs (cat. PAK1: sc-29700, PAK2: sc-26183, PAK3: sc-36181 Santa Cruz, Biotechnology, Inc.) using Lipofectamine 2000 (Life Technologies Co.). Cells were incubated with the liposome complexes for $20 \mathrm{~h}$, liposomes were aspirated, and fresh RPMI 1640 medium or DMEM containing 10\% FBS was added for the 2-3 h. Assays were performed $24 \mathrm{~h}$ after the beginning of transfection. PAK1, PAK2, and PAK3 knockdown was assessed by quantitative reverse transcription-PCR (qRT-PCR) (see below) $16 \mathrm{~h}$ after transfection and by western blot (WB) 24 and $48 \mathrm{~h}$ after transfection. Data are reported as the reduction in PAK1, PAK2, and PAK3 RNA by the siRNA versus scrambled sequence control.

\section{RNA isolation}

Cells were washed twice with PBS, and $800 \mu \mathrm{l}$ of TRIZOL (Life Technologies Co.) was added. Cells were scraped and transferred into $1.5 \mathrm{ml}$ tubes. To isolate RNA from human thyroid samples, the tissues were homogenized in $800 \mu \mathrm{l}$ of TRIZOL. After the addition of $200 \mu \mathrm{l}$ of chloroform, tubes were shaken, incubated for $2 \mathrm{~min}$ at room temperature, and centrifuged at $16000 \mathrm{~g}$ for $15 \mathrm{~min}$ at $4{ }^{\circ} \mathrm{C}$. The supernatant was transferred into a new $1.5 \mathrm{ml}$ tube, mixed with $400 \mu \mathrm{l}$ of isopropyl alcohol, and centrifuged at $16000 \mathrm{~g}$ for $15 \mathrm{~min}$ at $4{ }^{\circ} \mathrm{C}$. The supernatant was aspirated, and the pellets containing RNA were washed using $70 \%$ ethanol and air-dried. The RNA was reconstituted and concentration was measured by spectrophotometry.

\section{RT-PCR}

To examine PAK isoform expression in the thyroid cancer cell lines and human samples and to confirm PAK isoform-specific knockdown by siRNA, PAK isoform-specific RT-PCR was performed. To determine basal expression of PAK isoforms, PAKs 1-3 and PAK6 were amplified from cell lines using newly designed primers, while PAK4 and PAK5 were amplified with primers from Life Technologies Co. (Supplementary Table 2A, see section on supplementary data given at the end of this article). Identity was confirmed by amplicon size and melting curve analysis. For siRNA experiments, quantitative realtime RT-PCR using PAKs 1-3 sequence-specific primers and probes and Universal Master Mix (Life Technologies Co., Supplementary Table 2B, see section on supplementary data given at the end of this article) was performed. For all RT-PCRs, $440 \mathrm{ng}$ of RNA was treated with DNase I (Life Technologies Co.) for $15 \mathrm{~min}$, and $132 \mathrm{ng}$ of DNase-treated RNA was reverse transcribed using the TaqMan RT Reagents kit (Life Technologies Co.). PCR was performed in 96 sample plates using cDNA equivalent to $18 \mathrm{ng}$ of total RNA (4 $\mu \mathrm{l}$ of RT reaction mixture) per $25 \mu \mathrm{l}$ per well. To normalize PAK gene expression for quantitative experiments and to confirm RNA integrity for all experiments, 18S rRNA was amplified using Taqman Ribosomal RNA control reagents kit as previously described (Ringel et al. 2001). PCR was performed as follows: after initial $2 \mathrm{~min}$ incubation at $50{ }^{\circ} \mathrm{C}$ for inactivation of AmpErase UNG activity, the cDNA was denatured at $94{ }^{\circ} \mathrm{C}$ for $10 \mathrm{~min}$. The samples were then subjected to 40 cycles of a two-step amplification protocol that included $15 \mathrm{~s}$ at $94{ }^{\circ} \mathrm{C}$ and a $1 \mathrm{~min}$ annealing-elongation step at $60{ }^{\circ} \mathrm{C}$. 
PAKs 1-6 and 18S were amplified in all samples in duplicate in two separate reactions. Negative controls were included for the RT (RT negative) and PCRs (non-template control). To normalize data to $18 \mathrm{~S}$ control and to quantify results, we use the $\Delta C_{\mathrm{t}}$ formula as previously described (Ringel et al. 2001). All samples were electrophoresed to confirm the size and specificity.

\section{Protein extraction and western blotting}

Cells were washed twice with ice-cold PBS, scraped, and resuspended with $500 \mu \mathrm{l}$ of PBS. After centrifugation at $500 \mathrm{~g}$ for $5 \mathrm{~min}$, cells were lysed with M-PER buffer (Fisher Scientific, Pittsburgh, PA, USA) containing $0.3 \mu \mathrm{M}$ okadaic acid, $1 \mu \mathrm{g} / \mathrm{ml}$ of aprotinin, pepstatin, and leupeptin, and $20 \mathrm{mM}$ of 4-amidinophenyl methane-sulfonyl fluoride. After $10 \mathrm{~min}$ incubation with the M-PER buffer on ice, the lysate was centrifuged at $16000 \mathrm{~g}$ for $15 \mathrm{~min}$ at $4{ }^{\circ} \mathrm{C}$. Supernatant was collected, and protein concentrations were measured by BCA protein assay (Fisher Scientific).

Twenty-five micrograms of total lysate were suspended in reducing SDS buffer (Life Technologies Corporation) and boiled for $5 \mathrm{~min}$. The reduced and denatured lysate was loaded into 4-12\% SDS-PAGE, separated by electrophoresis, and transferred to nitrocellulose membranes, and immunoblotting was performed as described (Ringel et al. 2001, Vasko et al. 2007). Primary antibodies against PAK1 (\#2602), PAK2 (\#2608), PAK4 (\#2242), phospho-Thr423 PAK1/Thr402 PAK2 (\#2601), PAK1/2/3 (\#2604), cRAF (\#9422), and phospho-Ser338 cRAF (\#9427) were obtained from Cell Signaling Technology (Danvers, MA, USA). Antibodies against PAK3 (sc-1871), PAK6 (sc-32857), and $\alpha$-tubulin (sc-5546) were obtained from Santa Cruz Biotechnology, Inc. Anti-PAK5 antibody (st-1097) was obtained from EMD Biosciences (Gibbstown, NJ, USA). Antibody against GAPDH (NB300-327) was obtained from NOVUS Biologicals, Inc. (Littleton, CO, USA). Antibodies against vimentin (V6630) and phospho-Ser55 vimentin were obtained from Sigma-Aldrich, Inc. and MBL Co. (Nagoya, Japan) respectively.

\section{Migration assay}

Cancer cells were grown in DMEM and RPMI 1640 medium containing FBS until 30-40\% confluent, transfected, washed with PBS, trypsinized for 5-10 min, collected with 0\% FBS DMEM and RPMI 1640 medium, and centrifuged at $300 \mathrm{~g}$ for $5 \mathrm{~min}$. Cells were resuspended with 0\% FBS DMEM and RPMI 1640 medium and counted using a hemocytometer.
A volume of $10^{5}$ cells in $300 \mu$ medium was placed into upper chamber of Boyden chamber $(8 \mu \mathrm{m}$ pore $)$ inserts in 24-well plates filled with $400 \mu \mathrm{l}$ of either DMEM or RPMI 1640 medium containing 10\% FBS chemoattractant in the bottom chamber. Cells were incubated at $37{ }^{\circ} \mathrm{C}$ and $5 \% \mathrm{CO}_{2}$. The cells on and under the Boyden chamber membrane were fixed with $3.7 \%$ formaldehyde containing $0.05 \%$ crystal violet for 15 min after washing cells with PBS. The chambers were washed with distilled water and the excess water was eliminated. The cells on the top (non-migrated) and bottom (migrated) sides of the membrane were collected by scraping the top and bottom of the chamber with a Q-tip, which was subsequently placed into a $1.5 \mathrm{ml}$ tube. The remainder of the cells remained in the Boyden chamber. The Q-tips containing the scraped cells and the Boyden chamber containing the non-migrated cells were separately incubated in $80 \%$ methanol, shaken at $500 \mathrm{~g}$ for $30 \mathrm{~min}$, and the extracted dye was measured at $570 \mathrm{~nm}$. Migration was quantified as the ratio of the migrated cells over the total cells (non-migrated plus remaining cells) to calculate migration rates. Experiments were performed in duplicate on multiple occasions as described in the figures.

\section{Immunohistochemical staining}

Sections were dewaxed twice with xylene, soaked in 100 and $95 \%$ alcohol, and incubated in 3\% hydrogen peroxide for $15 \mathrm{~min}$ after microwave treatment in antigen unmasking solution (Vector Laboratories, Inc., Burlingame, CA, USA) for $10 \mathrm{~min}$. Sections were then incubated at $4{ }^{\circ} \mathrm{C}$ overnight with anti-PAK1 (dilution 1:100) and phospho-PAK (dilution 1:100) antibodies. Immunodetection was performed by the use of the Vectastain Universal Quick kit according to the manufacturer's instruction. Peroxidase staining was revealed in 3,3'-diaminobenzidine. Negative controls were performed as above except the primary antisera were not included. IHC was evaluated by three investigators independently ( $\mathrm{K} \mathrm{K} \mathrm{M,} \mathrm{M} \mathrm{S,} \mathrm{and}$ M D R) and qualitatively scored based on the intensity and number of positive cells.

\section{Statistical analysis}

As several of the experiments were conducted on different days, linear mixed effects models were used to take this into consideration. RT-PCR data were first normalized to internal control gene $18 \mathrm{~S}$ expression, and then linear mixed effects models were used for hypothesis testing. Variances of test statistics were estimated from a saturated model to ensure robust 
testing. For some simple two-group comparisons, paired $t$-tests were used. $P<0.05$ is considered as significant for single comparisons. Holm's procedure was used to adjust for multiple comparisons or multiple endpoints (Holm 1979) to strongly control type I error to $\alpha=0.05$.

\section{Results}

\section{PAKs 1-3, PAK4, and PAK6 are expressed in human thyroid cancer cell lines}

PAK isoform mRNA levels were measured in six confirmed human thyroid cancer cell lines (BCPAP, KTC1, TPC1, FTC133, C643, and SW1743) by RT-PCR using isoform-specific primers. All isoforms of PAK were expressed except for PAK5 in the cell lines (Fig. 1A). Similar to mRNA levels, PAK1, PAK2, PAK3, and PAK4 proteins were expressed in all six cell lines, while PAK5 was not detected on WB (Fig. 1B). PAK6 protein was identified at low levels in four of the six cell lines (not expressed in BCPAP and KTC1).

\section{Thyroid cancer cell motility is group I PAK-dependent}

The effect of PAK inhibition on cell migration was studied using Boyden chambers in which motility was induced by serum gradient. To determine the effect of group I PAKs, the six thyroid cancer cell lines were transfected with the group I PAK-specific PID inhibitor or a vector control. In all cell lines, transfection of the PID significantly decreased cell motility in the Boyden chambers (Fig. 2A; $P<0.05$ versus control transfection). To determine whether inhibition of PAK also reduced cell viability, 3-(4,5-dimethylthiazol-2-yl)2,5-diphenyltetrazolium bromide (MTT) assays were performed. No statistical significance of inhibition of cell viability was detected between the PID versus the control transfections for any cell line (Fig. 2B). To confirm that the PID blocked PAK activity, we examined the phosphorylation of two direct downstream targets of PAK, cRAF, and vimentin using antibodies that detect phosphorylation at PAK-specific sites in transfected FTC133 cells. WB confirmed that PAK phosphorylation of cRAF and vimentin was partially inhibited by PID to a degree consistent with the $\sim 50 \%$ transfection efficiency (Fig. 2C).

\section{Thyroid cancer cell migration is PAK1-dependent}

We next performed experiments to determine which of the group I PAK isoforms were involved in cell migration using siRNA. BCPAP and FTC133 cells
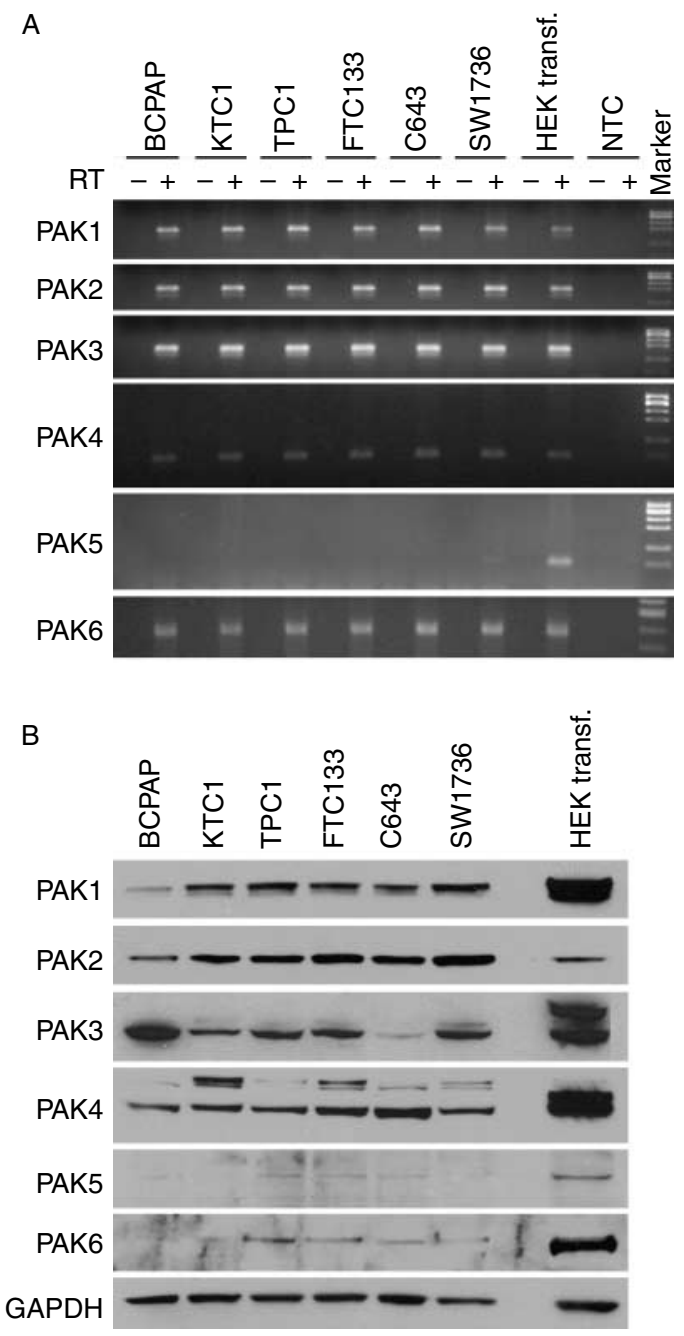

Figure 1 PAK isoform expression in thyroid cancer cells. Expression of PAK isoforms was examined by RT-PCR (A) and WB $(B)$ in six established human thyroid cancer cell lines.

(A) For the RT-PCR, negative controls were RT-negative reactions with RNA from each cell line and non-template control. The RNA from HEK293 cells transfected with PAK5 was used as a positive control, since HEK293 cells express all PAK isoforms except for PAK5. All PAK isoforms except PAK5 were expressed in all six human thyroid cancer cell lines. RT, reverse transcriptase; NTC, non-template control. (B) For WB, the protein was isolated from HEK293 cells transfected with each PAK isoform and used as positive controls. All six cell lines expressed all PAK1, PAK2, PAK3, and PAK4 isoforms, and no expression of PAK5 was seen. PAK6 protein was not detected in BCPAP and KTC1 cells.

were used because they are from the most common histological subtypes of thyroid cancer and express either mutant BRAF V600E or have PTEN loss respectively (Supplementary Table 1). PAK1 siRNA reduced transwell migration in the BCPAP and FTC133 cells (Fig. 3A; both $P<0.001$ versus scrambled sequence control). By contrast, no significant effect of the PAK2 or PAK3 siRNAs was 


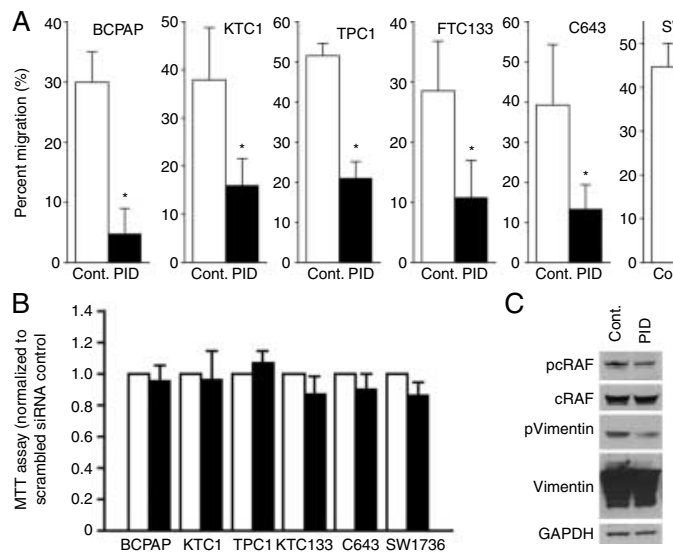

Figure 2 Group I PAKs regulate thyroid cancer cell migration. (A) All six thyroid cancer cell lines were transfected with GFP-tagged PID construct, a group I PAK-specific inhibitor. Transfection efficiencies were $\sim 40-60 \%$; PID transfection reduced migration by more than $50 \%$ compared to vector control in all six human thyroid cancer cell lines. * Signifies statistical significance; $P$ values were 0.0025 for BCPAP, 0.018 for KTC1, <0.0001 for TPC1, 0.0091 for FTC133, 0.0121 for C643, and 0.0054 for SW1736. (B) MTT assays were performed to examine cell viability. There was no difference between cells transfected with control vector and PID vector. (C) Phosphorylation status of two downstream targets of group I PAKs was examined by antibody against PAK-specific phosphorylation site using FTC133 cells. PID transfection reduced phosphorylation of both CRAF and vimentin without changing total protein levels to a level that corresponds with transfection efficiency.

identified. Specific reduction of the appropriate target PAK isoform mRNA and proteins was confirmed by real-time RT-PCR (Fig. 3B) $(\alpha=0.05$ controlled across PAK isoform comparisons within experiment using Holm's procedure) and WB (Fig. 3C) respectively. In some experiments, the PAK1 siRNA also reduced PAK2 protein levels (Fig. 3C), but the PAK2 siRNA was specific and had no effect on migration, suggesting that PAK1 is the primary isoform that regulates migration of the cell lines.

\section{Total and Thr423 phospho-PAK levels are increased in human PTC invasion}

We analyzed four frozen tumor and normal paired samples from human FTC and PTCs for PAK isoform expression by RT-PCR. Similar to the thyroid cancer cell lines, mRNA from all PAK isoforms was expressed with the exception of PAK5 (Fig. 4A). Because our prior gene expression data implicated PAK in the invasive fronts of PTCs, we used a second group of seven PTCs selected for the presence of invasion into local tissues for analysis by IHC using PAK1 and phospho-Thr423 PAK1/Thr402 PAK2 (pPAK) antibodies. The immunoactivity was heterogeneous in the tumors; however, clusters of invasive cells were characterized consistently by an increase in the levels of total PAK1 and pPAK compared to both the central and normal regions (representative data in Fig. 5). Overall, each individual evaluator determined that the invasive fronts were characterized by greater levels of immunoactive total PAK1 and pPAK, particularly in the clusters of cells invading through the capsule.

A
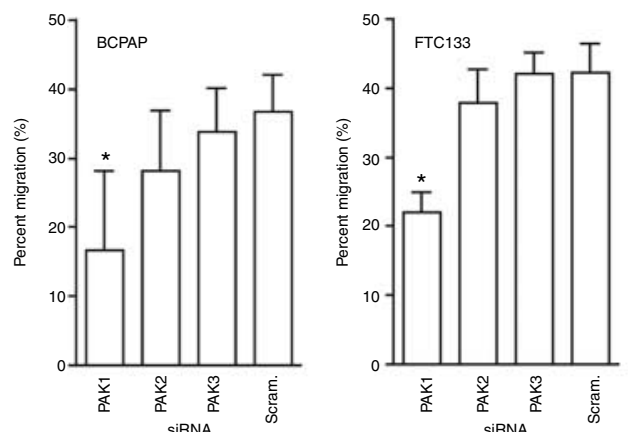

B BCPAP
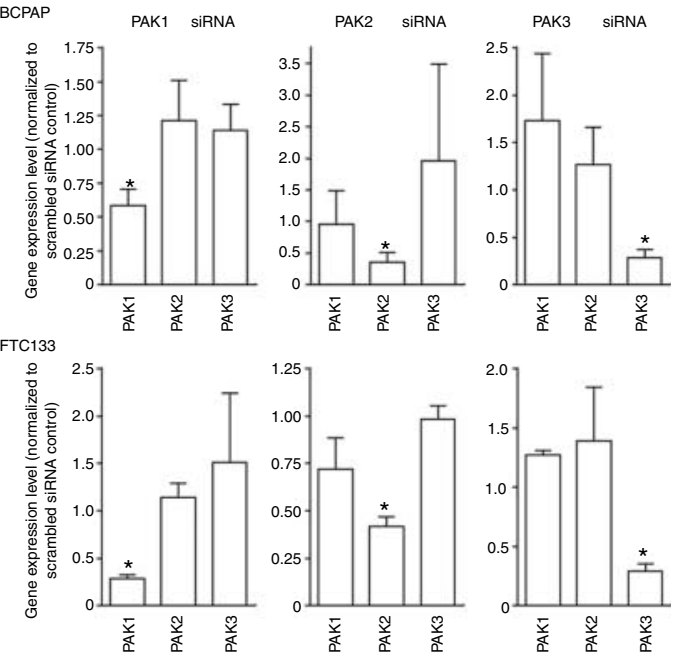

C
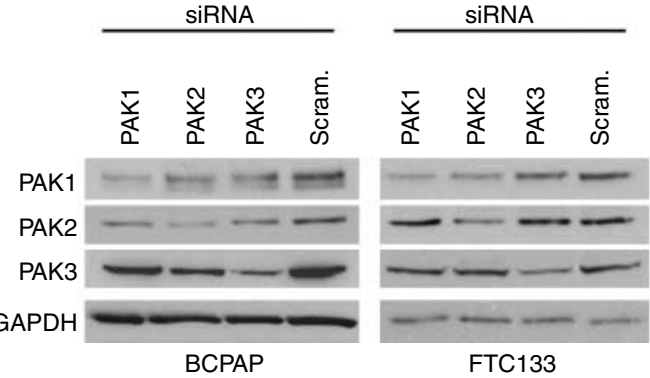

Figure 3 Thyroid cancer cell motility is PAK1-dependent. (A) Transfection of PAK1 siRNA reduced cell migration by about $50 \%$ compared to scramble control in BCPAP and FTC133 cells ( ${ }^{\star} P<0.01$ versus control). In contrast, there was no statistical significant change by the transfection of either PAK2- or PAK3-specific siRNA transfection in either cell line. (B and C) RT-PCR confirmed isoform-specific knockdown by RT-PCR (B: all $P<0.05$ ) and by western blot. 


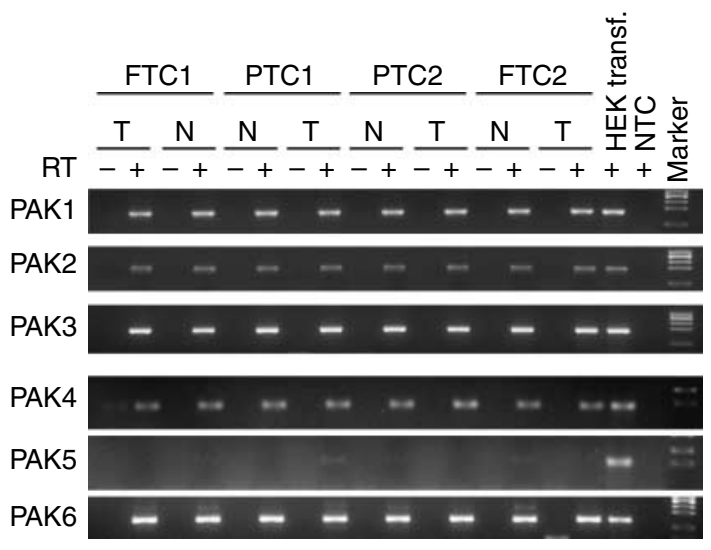

Figure 4 PAK mRNA levels in human thyroid tissues. Similar to cell line results, all six PAK isoform mRNAs were expressed in both normal thyroid and cancer tissues except PAK5. RNA from HEK293-transfected cells with PAK5 was used as a positive control. RT, reverse transcriptase; FTC, follicular thyroid cancer; PTC, papillary thyroid cancer; NTC, non-template control.

\section{Discussion}

In the present study, we have for the first time assessed PAK expression and function in thyroid cancer cell lines and clinical samples and provided data that support a functional role for PAKs in thyroid cancer cell biology. The rationale for this work was in part based on previously published work demonstrating a gene profile consistent with EMT in the invasive fronts of locally aggressive PTCs. Bioinformatics analysis of these data suggested that integrin signaling was up-regulated in the invasive fronts, and in vitro experiments demonstrated an association with PAKphosphorylated vimentin and cell invasion (Vasko et al. 2007). However, these studies did not directly assess PAK expression or activity in thyroid cancers and did not include functional studies of PAK in thyroid cancer cell lines.

\section{A}

PAK1

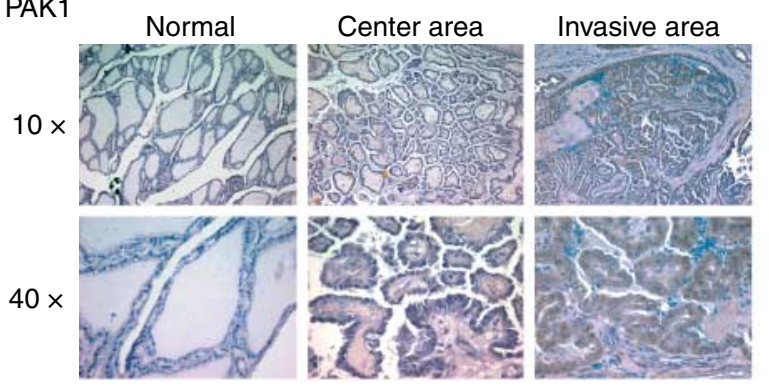

Human thyroid cancer cell lines, normal human thyroid tissue, and thyroid cancer tissue all express PAK1, PAK2, PAK3, PAK4, and in most cases PAK6. These patterns are similar to those found in other epithelial cells, such as breast and prostate (Kumar et al. 2006, Molli et al. 2009). PAK6 protein expression was more variable than the other isoforms in the thyroid cancer cell lines. This may be due to low levels of protein since the levels of PAK6 mRNA were lower than the other isoforms. It could also be related to post-translational degradation of PAKs or antibody sensitivity. Protein stability of PAK6 was not directly tested in this manuscript. Since the literature describes PAK1, PAK2, and PAK4 as regulators of cytoskeleton rearrangement and cell motility (Molli et al. 2009), and because PAK1 has been shown to be oncogenic in vivo (Wang et al. 2006), we focused these experiments on group I PAKs (PAK1, PAK2, and PAK3). We used a group I-specific molecular inhibitor, PID, which binds specifically to the inhibitory domain of group I PAKs and inhibits activation (Thullberg et al. 2007). Remarkably, the migration of each of the six thyroid cancer cell lines was inhibited by PID expression despite the difference in tumor types and genetic mutations. The migration inhibition was incomplete, likely related to the $\sim 50 \%$ transfection rate of the cells. Group I PAKs have also been shown to regulate cell proliferation (Molli et al. 2009). Expression of the PID did not affect thyroid cancer cell viability using MTT. It is possible that more subtle effects on cell proliferation would be detected using other methods; however, it is notable that the effect on migration was much more impressive in these same conditions. WB confirmed that phosphorylation of PAK substrates was reduced by transfection of the PID compared with control transfectants. While the data suggest an important role for group I PAKs in thyroid cancer cell

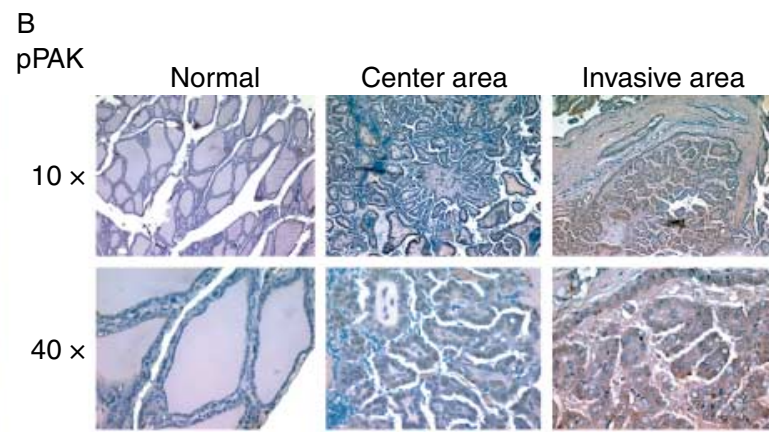

Figure 5 PAK1 and pPAK are overexpressed in PTC invasive fronts. Seven primary PTCs with local invasion from which sections were available containing normal thyroid, central tumor, and the invasive front on the same slide were selected for IHC for PAK1 and pPAK. Data from one representative sample are shown; $(A)$ is IHC for PAK1 at $10 \times$ and $40 \times$ power; $(B)$ is IHC for pPAK. Increased immunoactivity (brown staining) is noted in the invasive front. 
motility, an additional role for PAK4 or PAK6 in thyroid cells has not been excluded by these experiments.

Because all three group I PAKs are expressed in the thyroid cancer cells, we next sought to determine whether there was PAK isoform specificity for the anti-migration effect. For these experiments, we chose two of the confirmed thyroid cancer cell lines, BCPAP and FTC133. BCPAP has a BRAF V600E mutation, and FTC133 has loss of PTEN expression. In both cases, only the PAK1 siRNA significantly reduced migration compared with a scrambled sequence siRNA control. Isoform-specific reduction of PAK siRNA was demonstrated at the mRNA level by qRT-PCR. WB also demonstrated relative specificity of the siRNA, although there was some reduction in immunoactive PAK2 with the PAK1 siRNA due to either siRNA or antibody specificity. The RT-PCR data suggest the latter, and the absence of an effect of the PAK2 siRNA on migration supports the conclusion of a more important role for PAK1 in migration of the two cell lines. These results are also in concordance with breast cancer cell lines in which an increase in PAK1 has been seen in aggressive and invasive lines (Adam et al. 2000, Wang et al. 2006). The mechanisms for the greater impact of PAK1 loss compared with PAK2 or PAK3 require further investigation. While group I PAKs have been shown to have similar substrate specificities (Rennefahrt et al. 2007), in some cell systems unique effects have been reported, but the mechanisms and unique substrates require further study (Bright et al. 2009, Siu et al. 2010).

We next assessed the expression levels of PAK1 and PPAK in vivo. These data were performed using a different set of tumors that were used for microarray analysis in the prior studies and EMT protein staining that first implicated integrin signaling in thyroid cancer invasion (Vasko et al. 2007). To assess the level of PAK expression and activity in the invasive fronts, we performed IHC on this small series of PTCs that were selected for the presence of local invasion. In most cases, an increase in both PAK1 and PPAK was noted in the invasive front (representative case in Fig. 5). The staining in the tumors was heterogeneous with greatest levels of immunoactivity identified in the nests of tumor cells that were directly invading tumor capsules and local tissues. This heterogeneity is the rationale for focusing on IHC experiments rather than WB of frozen tumor, which in our tumor bank are from the central regions of the tumors. There are several potential reasons for the increase in PAK1 protein levels identified in the invasive fronts. These include both translational and post-translational mechanisms that require further study. In the present study, RNA was not available from the invasive fronts in the current study for direct comparison with protein levels. It is relevant to note that increased levels of PAK1 protein have also been demonstrated in colon cancer, and ovarian cancer has been associated with poor prognosis or poor prognostic features, suggesting this may be a common event in solid tumor progression (Carter et al. 2004, Siu et al. 2010).

In the clinical sample component of the studies, we recognize that the sample number is small, and in this series, we did not have adequate tissue to analyze for thyroid oncogene expression. However, in our prior study, expression of EMT-related proteins was associated with invasion and regional metastases independent of any particular thyroid oncogene (Vasko et al. 2007). Moreover, the inhibition of migration was consistent between the six cell lines despite being selected to express each of the most common thyroid oncogenes. These data together suggest that PAK is essential to maintaining the migratory capacity of motile thyroid cancer cells, and are consistent with the known key regulatory role of PAKs in regulating cytoskeletal dynamics and lamellapod formation (Bokoch 2003). PAKs are known to be regulated by several thyroid cancer-related signaling cascades including the phosphatidylinositol 3-kinase (PI3K), mitogen-activated protein kinase (MAPK), and Wnt pathways (Kumar et al. 2006). Further studies are ongoing to dissect the role of PAK in the action of these oncogenes in the context of thyroid cells with particular focus on MAPK and PI3K pathway interactions due to their roles in thyroid cancer. PAK is known to have complex interactions with members of both of these pathways. For example, PAK1 and AKT are both functional substrates of PDK1 (King et al. 2000) and interact with mammalian target of rapamycin (mTOR) (Ishida et al. 2007), and PAK1 physically binds and scaffolds AKT1 regulating its localization and activity (specific for AKT1; Higuchi et al. 2008). PAK1 also has been shown to phosphorylate and regulate MAPK pathway molecules including MEK and cRAF, and be important for oncogenemediated cell proliferation and MAPK activation (Nheu et al. 2004, Beeser et al. 2005, McDaniel et al. 2008, Somanath et al. 2009). It is recognized that in vivo PAK activity may be initiated by other secondary non-oncogene effectors including signaling molecules available in the tumor microenvironment. Finally, we have focused the current studies on the role of PAK activity in thyroid cancer tissues; however, 
some of the cell biology effects we have identified could be related to the scaffolding function of PAK1. The role for PAK1-mediated scaffolding in thyroid cancer cells is currently being studied.

In summary, we have defined the expression profiles of PAKs in human thyroid cancer cell lines and benign and malignant thyroid tissues. Functional studies demonstrate a broad role for group I PAKs in thyroid cancer cell migration and implicate PAK1 in this process. PAK1 expression and pPAK levels are enhanced in the invasive fronts in human thyroid cancer samples, consistent with the functional studies. Further research evaluating the scaffolding function of group I PAKs in this process and in identifying the critical upstream regulators of PAK is warranted to fully define the role of PAKs as potential mediators of thyroid cancer migration and invasion.

\section{Supplementary data}

This is linked to the online version of the paper at http://dx. doi.org/10.1677/ERC-10-0168.

\section{Declaration of interest}

The authors declare that there is no conflict of interest that could be perceived as prejudicing the impartiality of the research reported.

\section{Funding}

We would like to acknowledge grants from NIH (P01CA124570) to MDR that funded this work.

\section{Acknowledgements}

We would like to acknowledge the efforts of Rebecca Nagy, MS, CGC; Huiling $\mathrm{He}, \mathrm{PhD}$, and Paul Wakely, MD, in obtaining the frozen tissues for PAK mRNA analysis.

\section{References}

Adam L, Vadlamudi R, Mandal M, Chernoff J \& Kumar R 2000 Regulation of microfilament reorganization and invasiveness of breast cancer cells by kinase dead p21-activated kinase-1. Journal of Biological Chemistry 275 12041-12050. (doi:10.1074/jbc.275.16. 12041)

Beeser A \& Chernoff J 2005 Production and use of a cell permeable inhibitor of group A Paks (TAT-PID) to analyze signal transduction. Methods 37 203-207. (doi:10.1016/j.ymeth.2005.05.017)

Beeser A, Jaffer ZM, Hofmann C \& Chernoff J 2005 Role of group A p21-activated kinases in activation of extracellular-regulated kinase by growth factors. Journal of Biological Chemistry 280 36609-36615. (doi:10.1074/jbc.M502306200)

Bokoch GM 2003 Biology of the p21-activated kinases. Annual Review of Biochemistry 72 743-781. (doi:10. 1146/annurev.biochem.72.121801.161742)

Bright MD, Garner AP \& Ridley AJ 2009 PAK1 and PAK2 have different roles in HGF-induced morphological responses. Cellular Signalling 21 1738-1747. (doi:10. 1016/j.cellsig.2009.07.005)

Carter JH, Douglass LE, Deddens JA, Colligan BM, Bhatt TR, Pemberton JO, Konicek S, Hom J, Marshall M \& Graff JR 2004 Pak-1 expression increases with progression of colorectal carcinomas to metastasis. Clinical Cancer Research 10 3448-3456. (doi:10.1158/1078-0432.CCR03-0210)

Fabien N, Fusco A, Santoro M, Barbier Y, Dubois PM \& Paulin C 1994 Description of a human papillary thyroid carcinoma cell line. Morphologic study and expression of tumoral markers. Cancer 73 2206-2212. (doi:10.1002/ 1097-0142(19940415)73:8<2206::AID-CNCR2820730 $828>3.0 . \mathrm{CO} ; 2-\mathrm{M})$

Frost JA, Swantek JL, Stippec S, Yin MJ, Gaynor R \& Cobb MH 2000 Stimulation of NFKB activity by multiple signaling pathways requires PAK1. Journal of Biological Chemistry 275 19693-19699. (doi:10.1074/ jbc.M909860199)

Goretzki PE, Frilling A, Simon D \& Roeher HD 1990 Growth regulation of normal thyroids and thyroid tumors in man. Recent Results in Cancer Research $\mathbf{1 1 8}$ $48-63$.

Gustavsson B, Hermansson A, Andersson AC, Grimelius L, Bergh J, Westermark B \& Heldin NE 1996 Decreased growth rate and tumour formation of human anaplastic thyroid carcinoma cells transfected with a human thyrotropin receptor cDNA in NMRI nude mice treated with propylthiouracil. Molecular and Cellular Endocrinology 121 143-151. (doi:10.1016/03037207(96)03859-2)

Higuchi M, Onishi K, Kikuchi C \& Gotoh Y 2008 Scaffolding function of PAK in the PDK1-Akt pathway. Nature Cell Biology 10 1356-1364. (doi:10.1038/ ncb1795)

Holm S 1979 A simple sequentially rejective multiple test procedure. Scandinavian Journal of Statistics 6 65-70.

Holm C, Rayala S, Jirstrom K, Stal O, Kumar R \& Landberg G 2006 Association between Pak1 expression and subcellular localization and tamoxifen resistance in breast cancer patients. Journal of the National Cancer Institute 98 671-680. (doi:10.1093/jnci/djj185)

Ishida H, Li K, Yi M \& Lemon SM 2007 p21-activated kinase 1 is activated through the mammalian target of rapamycin/p70 S6 kinase pathway and regulates the replication of hepatitis $\mathrm{C}$ virus in human hepatoma cells. Journal of Biological Chemistry 282 11836-11848. (doi:10.1074/jbc.M610106200) 
Jaffer ZM \& Chernoff J 2002 p21-activated kinases: three more join the Pak. International Journal of Biochemistry and Cell Biology 34 713-717. (doi:10.1016/S13572725(01)00158-3)

Kalluri R \& Weinberg RA 2009 The basics of epithelialmesenchymal transition. Journal of Clinical Investigation 119 1420-1428. (doi:10.1172/JCI39104)

King CC, Gardiner EM, Zenke FT, Bohl BP, Newton AC, Hemmings BA \& Bokoch GM 2000 p21-activated kinase (PAK1) is phosphorylated and activated by 3-phosphoinositide-dependent kinase-1 (PDK1). Journal of Biological Chemistry 275 41201-41209. (doi:10.1074/jbc.M006553200)

Kumar R \& Vadlamudi RK 2002 Emerging functions of p21-activated kinases in human cancer cells. Journal of Cellular Physiology 193 133-144. (doi:10.1002/jcp. 10167)

Kumar R, Gururaj AE \& Barnes CJ 2006 p21-activated kinases in cancer. Nature Reviews. Cancer 6 459-471. (doi:10.1038/nrc1892)

Kurebayashi J, Tanaka K, Otsuki T, Moriya T, Kunisue H, Uno M \& Sonoo H 2000 All-trans-retinoic acid modulates expression levels of thyroglobulin and cytokines in a new human poorly differentiated papillary thyroid carcinoma cell line, KTC-1. Journal of Clinical Endocrinology and Metabolism 85 2889-2896. (doi:10. 1210/jc.85.8.2889)

Leboulleux S, Rubino C, Baudin E, Caillou B, Hartl DM, Bidart J-M, Travagli J-P \& Schlumberger M 2005 Prognostic factors for persistent or recurrent disease of papillary thyroid carcinoma with neck lymph node metastases and/or tumor extension beyond the thyroid capsule at initial diagnosis. Journal of Clinical Endocrinology and Metabolism 90 5723-5729. (doi:10.1210/ jc.2005-0285)

McDaniel AS, Allen JD, Park SJ, Jaffer ZM, Michels EG, Burgin SJ, Chen S, Bessler WK, Hofmann C, Ingram DA et al. 2008 Pak1 regulates multiple c-kit mediated RasMAPK gain-in-function phenotypes in $\mathrm{Nf1}+/-$ mast cells. Blood 112 4646-4654. (doi:10.1182/blood-200804-155085)

Molli PR, Li DQ, Murray BW, Rayala SK \& Kumar R 2009 PAK signaling in oncogenesis. Oncogene 28 2545-2555. (doi:10.1038/onc.2009.119)

Nheu T, He H, Hirokawa Y, Walker F, Wood J \& Maruta H 2004 PAK is essential for RAS-induced upregulation of cyclin D1 during the $\mathrm{G}_{1}$ to $\mathrm{S}$ transition. Cell Cycle $\mathbf{3}$ 71-74.

Pulcrano M, Boukheris H, Talbot M, Caillou B, Dupuy C, Virion A, De Vathaire F \& Schlumberger M 2007 Poorly differentiated follicular thyroid carcinoma: prognostic factors and relevance of histological classification. Thyroid 17 639-646. (doi:10.1089/thy.2007.0029)

Rayala SK, Molli PR \& Kumar R 2006 Nuclear p21-activated kinase 1 in breast cancer packs off tamoxifen sensitivity. Cancer Research 66 5985-5988. (doi:10.1158/00085472.CAN-06-0978)
Rennefahrt UEE, Deacon SW, Parker SA, Devarajan K, Beeser A, Chernoff J, Knapp S, Turk BE \& Peterson JR 2007 Specificity profiling of Pak kinases allows identification of novel phosphorylation sites. Journal of Biological Chemistry 282 15667-15678. (doi:10.1074/ jbc.M700253200)

Ringel MD 2009 Molecular markers of aggressiveness of thyroid cancer. Current Opinion in Endocrinology, Diabetes, and Obesity 16 361-366. (doi:10.1097/MED. 0b013e32832ff2cb)

Ringel MD, Hayre N, Saito J, Saunier B, Schuppert F, Burch H, Bernet V, Burman KD, Kohn LD \& Saji M 2001 Overexpression and overactivation of Akt in thyroid carcinoma. Cancer Research 61 6105-6111.

Schlumberger M \& Sherman SI 2009 Clinical trials for progressive differentiated thyroid cancer: patient selection, study design, and recent advances. Thyroid 19 1393-1400. (doi:10.1089/thy.2009.1603)

Schweppe RE, Klopper JP, Korch C, Pugazhenthi U, Benezra M, Knauf JA, Fagin JA, Marlow L, Copland JA, Smallridge RC et al. 2008 Deoxyribonucleic acid profiling analysis of 40 human thyroid cancer cell lines reveals cross-contamination resulting in cell line redundancy and misidentification. Journal of Clinical Endocrinology and Metabolism 83 4331-4341. (doi:10. 1210/jc.2008-1102)

Siu MK, Wong ES, Chan HY, Kong DS, Woo NW, Tam KF, Ngan HY, Chan QK, Chan DC, Chan KY et al. 2010 Differential expression and phosphorylation of Pak1 and Pak2 in ovarian cancer: effects on prognosis and cell invasion. International Journal of Cancer 127 21-31. (doi:10.1002/ijc.25005)

Somanath PR, Vijai J, Kichina JV, Byzova T \& Kandel ES 2009 The role of PAK-1 in activation of MAP kinase cascade and oncogenic transformation by Akt. Oncogene 28 2365-2369. (doi:10.1038/onc.2009.114)

Tanaka J, Ogura T, Sato H \& Hatano M 1987 Establishment and biological characterization of an in vitro human cytomegalovirus latency model. Virology 161 62-72. (doi:10.1016/0042-6822(87)90171-1)

Thiery JP 2002 Epithelial-mesenchymal transitions in tumour progression. Nature Reviews. Cancer 2 442-454. (doi:10.1038/nrc822)

Thullberg M, Gad A, Beeser A, Chernoff J \& Stromblad S 2007 The kinase-inhibitory domain of p21-activated kinase 1 (PAK1) inhibits cell cycle progression independent of PAK1 kinase activity. Oncogene 26 1820-1828. (doi:10.1038/sj.onc.1209983)

Tsuji T, Ibaragi S \& Hu G-F 2009 Epithelial-mesenchymal transition and cell cooperativity in metastasis. Cancer Research 69 7135-7139. (doi:10.1158/0008-5472.CAN09-1618)

Vadlamudi RK \& Kumar R 2003 P21-activated kinases in human cancer. Cancer Metastasis Reviews 22 385-393. (doi:10.1023/A:1023729130497) 
Vasko VV \& Saji M 2007 Molecular mechanisms involved in differentiated thyroid cancer invasion and metastasis. Current Opinion in Oncology 19 11-17. (doi:10.1097/ CCO.0b013e328011ab86)

Vasko V, Espinosa AV, Scouten W, He H, Auer H, Liyanarachchi S, Larin A, Savchenko V, Francis GL, de la Chapelle A et al. 2007 Gene expression and functional evidence of epithelial-to-mesenchymal transition in papillary thyroid carcinoma invasion. PNAS 104 2803-2808. (doi:10.1073/pnas.0610733104)

Wang RA, Zhang H, Balasenthil S, Medina D \& Kumar R 2006 PAK1 hyperactivation is sufficient for mammary gland tumor formation. Oncogene $\mathbf{2 5}$ 2931-2936. (doi:10.1038/sj.onc.1209309)
Xu X, Quiros RM, Gattuso P, Ain KB \& Prinz RA 2003 High prevalence of BRAF gene mutation in papillary thyroid carcinomas and thyroid tumor cell lines. Cancer Research 63 4561-4567.

Zhao ZS \& Manser E 2005 PAK and other Rho-associated kinases - effectors with surprisingly diverse mechanisms of regulation. Biochemical Journal 386 201-214. (doi:10. 1042/BJ20041638)

Zhao L, Ma QL, Calon F, Harris-White ME, Yang F, Lim GP, Morihara T, Ubeda OJ, Ambegaokar S, Hansen JE et al. 2006 Role of p21-activated kinase pathway defects in the cognitive deficits of Alzheimer disease. Nature Neuroscience 9 234-242. (doi:10.1038/ nn1630) 